\title{
Design of a Lebanese Cube Satellite ${ }^{\dagger}$
}

\author{
Ali J. Ghandour 1,* and Mohamad Jaafar Abdallah ${ }^{2}$ \\ 1 National Council for Scientific Research, Zahia Salmane street, Jnah, Beirut 11-8281, Lebanon \\ 2 Islamic University of Lebanon, Beirut 30014, Lebanon; m-abdallah@live.com \\ * Correspondence: aghandour@cnrs.edu.lb \\ + Presented at the 2nd International Electronic Conference on Remote Sensing, 22 March-5 April 2018; \\ Available online: https://sciforum.net/conference/ecrs-2.
}

Published: 22 March 2018

\begin{abstract}
Nowadays, nanosatellites are widely used in space technology due to their small size, ease of deployment, and relatively short development period. CubeSat specifications have been suggested as an effort to standardize nanosatellite mission design. Standardization opens the door for inter-CubeSat communications that can be used to form a CubeSat Cloud and mimic regular large multifunctional satellites with wide range of features, measurements, and sensing capabilities. In this paper, we introduce a Comprehensive CubeSat (CoCube, Gurgaon, India) online database. CoCube database focuses mainly on the different subsystems used during the design and implementation stages of existing CubeSat missions. Based on the lessons learned by comparing various CubeSat design alternatives and components' structures and analyzing the best practices of CubeSat development, LibanSAT design is introduced. LibanSAT is a 1U CubeSat that serves two main objectives: (i) greenhouse gases observation and (ii) educational purposes. We benchmarked off-the-shelf subsystems from various suppliers and chose the most suitable for our target mission based on cost, size, weight, and power consumption. Finally, we introduce a new CubeSat security algorithm based on predefined anomaly detection baseline that serves as intrusion prevention system for the control channel.
\end{abstract}

Keywords: nanosatellites; CubeSat; satellite security; greenhouse gases observation

\section{Introduction}

Designed using accessible off-the shelf technology, nanosatellites technology has drawn significant attention in research communities and industrial sectors. This is mainly due to its small size that does not compromise the needed performance. A nanosatellite development period is relatively short, which results in a reduction of the overall cost budget [1].

In order to standardize the design of nanosatellites mission, and develop the skills necessary for manufacturing and testing nanosatellites, CubeSat Design specification (CDS) [2] has been suggested [2]. Satellites are usually classified based on mass, but volume is the critical factor in the case of CubeSats. A unit referred to as " $U$ " is introduced: 1U CubeSat is a satellite with maximum mass of $1.33 \mathrm{~kg}$ and typical size of $10 \times 10 \times 10 \mathrm{~cm}$. Initially, CDS included standards for 1 to $3 \mathrm{U}$ designs only where $3 \mathrm{U}$ can be defined as a triple $1 \mathrm{U}$ CubeSat. The need for larger CubeSat missions required a CDS extension to provide $6 \mathrm{U}$ to $12 \mathrm{U}$ standard specification.

The contribution of this paper is threefold: (i) First, we introduce a database of all existing CubeSat missions with a focus on different subsystems used during the design and implementation phases; (ii) Based on the lessons learned from previous missions' database, we designed LibanSat, the first CubeSat for Lebanon. This is a $1 \mathrm{U}$ CubeSat built using ready-to-use off-the-shelfcomponents. (iii) Finally, the third contribution is to introduce a new CubeSat communication 
security algorithm based on predefined anomaly detection that serves as intrusion prevention system for the control channel.

The rest of the paper is organized as follows: Section 2 elaborates on the Comprehensive CubeSat database (CoCube, Gurgaon, India) schema. LibanSat primary and secondary goals are discussed in Section 3, in which the proposed mission design is introduced. A novel CubeSat communication security algorithm is depicted in Section 4. Finally, Conclusion is given in Section 5.

\section{CoCube Database}

Our first contribution in this manuscript is the design of a database, referred to as Comprehensive CubeSat database (CoCube, Gurgaon, India). Several existing CubeSat databases are published online [2-9]. However, these databases lack precise and updated information about the compiled missions. More importantly, existing databases do not discuss or contain relevant information about the subsystems used for each mission design. Subsystems details can be very useful for research in this field working towards the design of their own missions. CoCube database is mainly divided into three sections, as shown in Figure 1. Each section is composed of many tables, as discussed in the following points:

- Design Section: contains basic data related to missions under design, or waiting for launch opportunities, or currently deployed or decommissioned, and is made of the following three tables:

- Basic Info Table: such as size and mission goal.

- Provider Table: such as provider details and the country of origin.

- References Table: useful sources related to the mission.

- Launch Section: contains one table that includes all launch related details. This section is restricted to deployed and decommissioned missions only.

- Subsystems Section: represents the main contribution of the CoCube database as none of the published databases contains subsystems related data as far as the authors know. Each subsystem is tabulated alone with respective components and characteristics.

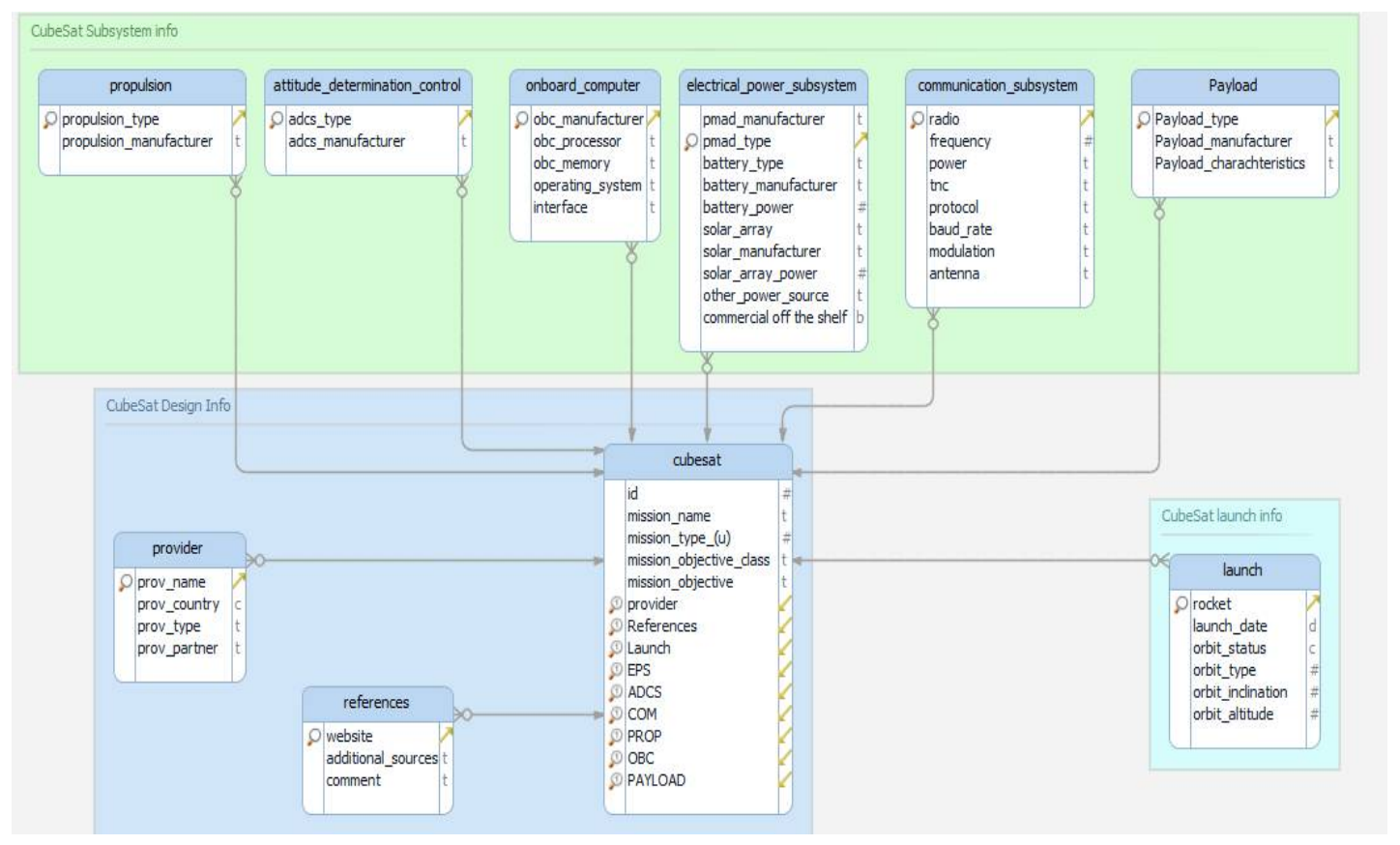

Figure 1. CoCube database schema. 


\section{LibanSat Mission}

\subsection{Mission Goals}

Nowadays, developing countries are able to design their own CubeSats using ready-to-use Component Off The Shelf (COTS). In this manuscript, we introduce LibanSat, a 1U CubeSat that serves as a remote sensing unit aimed at detecting greenhouse gases emissions. Greenhouse gas detection is set as the primary objective for the proposed LibanSat mission, whereas the second objective is an educational one. Data generated from our mission will be published and made available online to contribute towards the international effort to monitor and reduce greehouse gases emission.

In addition to its primary mission goal, LibanSat has an educational secondary purpose. After getting a launch opportunity for our CubeSat, we will transfer the experience gained from this project to local researchers and graduate students through presentations and workshops. In addition to that, we are designing a graduate course module in this field where we will demo a CubeSat prototype we built using Raspberry Pi and low cost equipment.

\subsection{Mission Design}

The main subsystems typically used in a CubeSat architecture are the following:

- $\quad$ Structure

- Payload

- Onboard Computer (OBC).

- $\quad$ Electrical Power System (EPS):

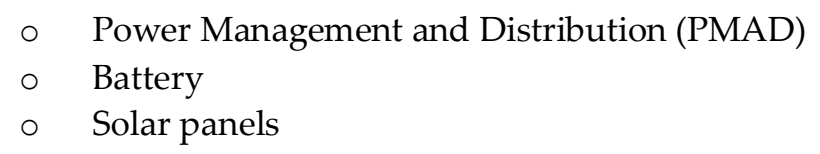

- $\quad$ Communication subsystem (COM)

- Attitude Determination and Control System (ADCS)

We will not elaborate on each of these subsystems due to space limitation, but instead we will focus on the payload. Argus-1000 spectrometer functions in the near infrared spectrum and can be effectively used for greenhouse gases detection with a matching mass of $230 \mathrm{~g}$ and a suitable volume of $45 \mathrm{~mm} \times 50 \mathrm{~mm} \times 80 \mathrm{~mm}$. In fact, two CubeSat missions, Canx-2 and SNSAT [5], sucessfully used the Argus-1000 in the outer space. Our contribution lies in the fact that this is the first attempt to the best of our knowledge to include this sensor in a $1 \mathrm{U}$ CubeSat.

In order to choose the remaining subsystems suitable for our mission, and especially our payload, we compare subsystems offered by different companies and choose the most appropriate ones. Due to space limitations, we will not show the detailed comparison matrix, and only selected subsystems are shown in the Table 1 with correspondent characteristics.

The EPS cost includes all needed power parts, including batteries and solar panels. We choose solar panels from ISIS, as they provide solar panels with antenna holder. Communication subsystem is chosen from GomSpace, as it ensures the best baud rate, and the lightest shape in the market, with an acceptable power consumption. A3200 OBC is more expensive than other options in the market; however, mass, volume, and power consumption were higher priorities in our design. The chosen OBC serves also as ACDS, as it contains 3-Axis magneto resistive sensor and 3-Axis gyroscope. The ground station is provided by GomSpace too to ensure utmost compatibility with the communication subsystem, knowing that it had the best price in the market. Please note that we do not refer to the ACDS in Table 1, as we do not use a standalone subsystem for it, and instead we rely on the solar panel with sun sensors, temperature sensors, and magnetorquer and OBC sensors.

Mass and volume budget shown at the end of Table 1 shows a good margin that can be used for wiring and buses, in addition to any future change in the design. It is clear that the payload constitutes around $49 \%$ of the cost of the proposed CubeSat design. Selected solar panels deliver 
around $2.3 \mathrm{~W}$, which is aligned with our power consumption budget that is around $1 \mathrm{~W}$ calculated with $20 \%$ contingency. Finally, the 22 Whr battery will be suitable for the eclipse time.

Table 1. Mass, height, cost, and power budgets of proposed LibanSat components [10-13].

\begin{tabular}{|c|c|c|c|c|c|c|c|c|}
\hline Subsystem & Manufacturer & Module & $\begin{array}{c}\text { Mass } \\
\text { (g) }\end{array}$ & $\begin{array}{c}\text { Height } \\
\text { (mm) }\end{array}$ & $\begin{array}{c}\text { Power } \\
(\mathrm{mW})\end{array}$ & $\begin{array}{c}\text { Duty Cycle } \\
(\%)\end{array}$ & $\begin{array}{l}\text { Power } \\
(\mathrm{mW})^{1}\end{array}$ & $\begin{array}{c}\text { Cost } \\
(€)\end{array}$ \\
\hline Structure & ISIS Inc. & & 87.2 & & & & & 2500 \\
\hline EPS & Crystal Space & $\mathrm{P} 1 \mathrm{u}$ & 420 & 13 & 15 & 100 & 18 & 16,000 \\
\hline \multirow{2}{*}{$\mathrm{COM}$} & \multirow{2}{*}{ GomSpace } & \multirow{2}{*}{$\mathrm{AX} 100$} & \multirow{2}{*}{24.5} & \multirow{2}{*}{6.5} & $2640^{2}$ & 3 & 95 & \multirow{2}{*}{6000} \\
\hline & & & & & $180^{3}$ & 100 & 216 & \\
\hline OBC & GomSpace & A 3200 & 24 & 7.1 & 130 & 100 & 156 & 6000 \\
\hline Antenna & ISIS Inc. & & 100 & & & & & 5000 \\
\hline Payload & Thoth tech. & $\begin{array}{c}\text { Argus } \\
1000\end{array}$ & 230 & 45 & 2300 & 20 & 552 & 53,000 \\
\hline $\begin{array}{l}\text { Ground } \\
\text { station }\end{array}$ & GomSpace & & & & & & & 38,500 \\
\hline & Total & & 888 & 71.6 & & & 1050 & 127,000 \\
\hline & Standard limit & & 1330 & 100 & & & & \\
\hline & Margin & & -442 & -28.4 & & & & \\
\hline
\end{tabular}

${ }^{1}$ Power consumed during duty cycle with $20 \%$ contingency; ${ }^{2}$ Power consumed during transmission;

${ }^{3}$ Power consumed during reception.

\section{CubeSat Security}

The last contribution of this manuscript is the security subsystem. An anomaly-based intrusion prevention system is designed and implemented for LibanSat mission. Satellites, like any other wireless technology, face some risks and vulnerabilities. In order to understand the security threats of a system, it is important to understand the way it communicates. In our case, the communication subsystem is the first critical part that must be taken into consideration. Although in the present time CubeSats might not be a priority target for hackers and terrorists, the rapid evolution of this technology may increase this threat.

CubeSats have two types of communication channels: (i) data channel and (ii) control channel. Data channel is a downward directional communication link in which data gathered by sensors and payloads is sent from the satellite to the ground station. Data encryption algorithms can be utilized for critical data, especially for military missions. However, in the majority of the cases, data gathered is not sensitive. Control channel is critical, since it is used for controlling satellite from the ground. CubeSat Space Protocol (CSP) [14], which is a small network/transport layer protocol designed by Aalborg University, is widely used nowadays in CubeSat missions design, and hence the proposed security algorithm will be implemented on top of this protocol.

Majority of CubeSat OBC is based on Linux or FreeRTOS open source operating system [15]. In some missions, we even witnessed the use of a smartphone as an $\mathrm{OBC}$ [16]. The main threat comes from the fact that once launched, operating systems are no longer updated with regularly released security patches due to tight link capacity.

To cope with this problem, we propose a new security protocol for our CubeSat. Due to the power and mass limitations, we introduced a lightweight Intrusion Prevention System (IPS) based on predefined anomaly. We define a baseline state as a network's traffic load, protocol used, and regular package size. In addition, based on orbit parameter, we can easily predict the pass time of CubeSat over corresponding ground station, and thus we use this value in the predefined baseline state. We also use the location of the CubeSat in case of onboard existing GPS module. Any received packet will be monitored by the anomaly detection agent. In the case of baseline misuse, the anomaly engine will drop the packet and $\log$ it for future forensics. After five misuses, transmitter will be added to our block list, and baseline will be updated accordingly. The block diagram of the proposed algorithm is shown in Figure 2. 


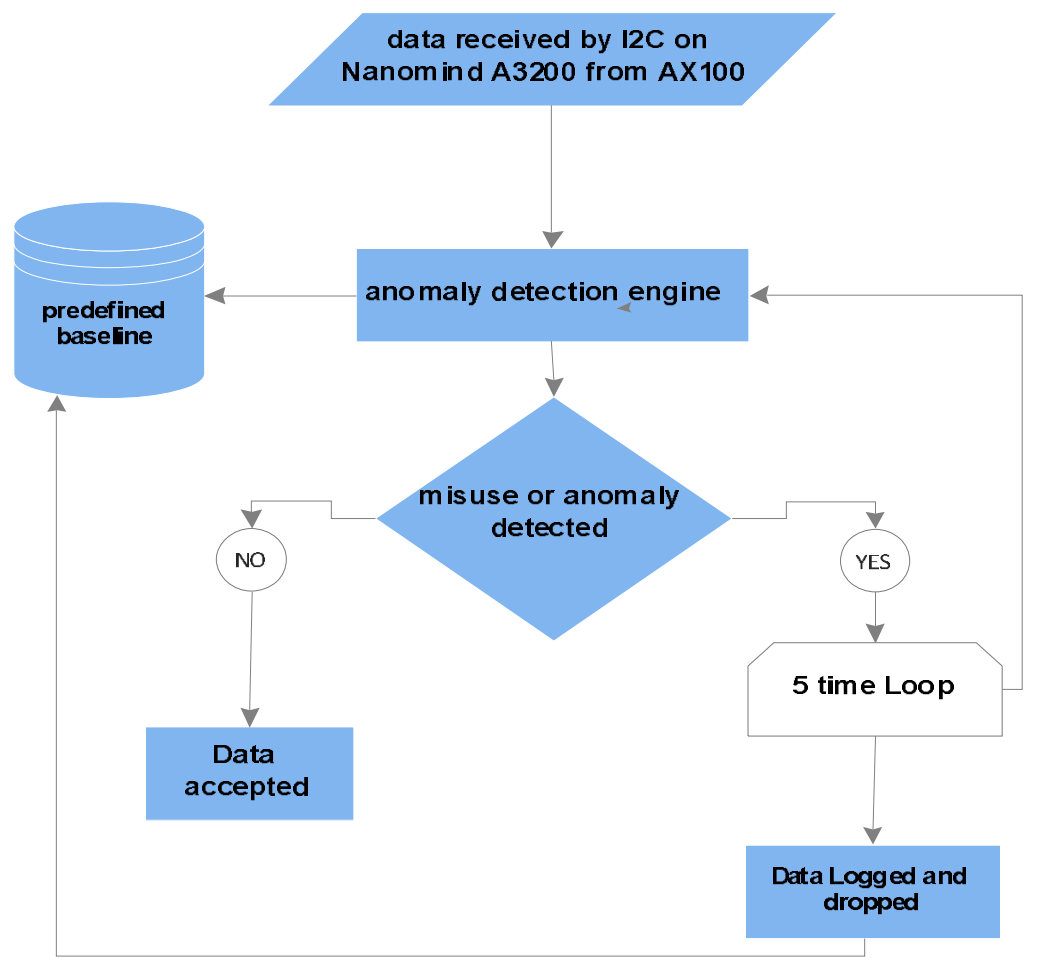

Figure 2. Flow chart of prosed anomaly detection algorithm.

\section{Conclusions}

In this paper, we introduce LibanSat, the first Lebanese 1U CubeSat. LibanSat design is based on learned lessons from Comprehensive CubeSat database that focuses on the subsystems used during the design and implementation phases of previous CubeSat missions. A security algorithm is suggested based on a lightweight intrusion prevention system that does not compromise CubeSat performance. In future work, we will expand the proposed security algorithm with better detection techniques and overhead simulation.

Conflicts of Interest: The authors declare no conflict of interest.

\section{References}

1. National Academies of Sciences, Engineering, and Medicine. Achieving Science with CubeSats: Thinking Inside the Box; The National Academies Press: Washington, DC, USA, 2016; doi:10.17226/23503.

2. CubeSat. Available online: http://www.cubesat.org (accessed on 5 April 2017); Gunter Space. Available online: http://space.skyrocket.de (accessed on 7 January 2017).

3. NanoSat. Available online: http://www.nanosats.eu (accessed on 6 January 2017).

4. CubeSat Database. Available online: https://sites.google.com/a/slu.edu/swartwout/home/cubesatdatabase (accessed on 3 January 2017).

5. EoPortal Directory. Available online: https://directory.eoportal.org (accessed on 4 January 2017).

6. Poghosyan, A.; Golkar, A. CubeSat evolution: Analyzing CubeSat capabilities for conducting science. Prog. Aerosp. Sci. 2017, 88, 59-83.

7. Klofas, B.; Anderson, J.; Leveque, K. 5th Annual CubeSat Developers Workshop; 5th Annual CubeSat Developers Workshop: San Luis Obispo, CA, USA, 2008.

8. CubeSats Satellites. Available online: https://www.n2yo.com (accessed on 6 March 2017).

9. Polat, H.C.; Josep, V.; Marcello, R. Survey, Statistical Analysis and Classification of Launched CubeSat Missions with Emphasis on the Attitude Control. J. Small Satell. 2016, 5, 513-530.

10. Argus 1000 User Manual. Available online: http://www.thothx.com (accessed on 2 April 2017).

11. Innovative Solutions in Space. Available online: https://www.isispace.nl (accessed on 20 March 2017).

12. CubesatShop. Available online: https://www.cubesatshop.com (accessed on 21 March 2017).

13. GomSpace. Available online: https://gomspace.com (accessed on 25 March 2017). 
14. CubeSat Space Protocol. Available online: https://github.com/GomSpace/ (accessed on 4 April 2017).

15. Paternina-Anaya, J.J.; Salamanca-Céspedes, J.E.; Ávila-Angulo, M.A. Communication system design and implementation for one pico-Satellite and four earth stations using AX.25. In Proceedings of the 2014 IEEE Central America and Panama Convention (CONCAPAN XXXIV), Panama City, Panama, 12-14 November 2014; doi:10.1109/concapan.2014.7000426.

16. Beukelaers, V.; Boshuizen, C.; Guillen, A.; Howard, B.; Marshall, W.; Safyan, M.; Tintore, O. PhoneSat 2.0. In Proceedings of the Fourth European CubeSat Symposium, ERM (Ecole Royale Militaire), Brussels, Belgium, 30 January-1 February 2012.

(C) 2018 by the authors. Licensee MDPI, Basel, Switzerland. This article is an open access article distributed under the terms and conditions of the Creative Commons Attribution (CC BY) license (http://creativecommons.org/licenses/by/4.0/). 